\title{
Experimental Compressor Multidisciplinary Optimization Using Different Parameterization Schemes
}

\author{
Igor Egorov ${ }^{1}$, Tatiana Buyukli ${ }^{2}$, Grigorii Popov ${ }^{3}$, Evgeni Goriachkin ${ }^{3}$, Yulia Novikova $^{3}$ and Andrey Volkov ${ }^{3}$ \\ ${ }^{1}$ Deputy General Designer, Lyulka Design Bureau, Moscow, Russia \\ 2Department of Technologies of Aircraft GTE Modeling, Central Institute of Aviation Motors Named after P.I. Baranov, Moscow, Russia \\ ${ }^{3}$ Department of Aircraft Engines Theory, Samara University, Samara, Russia
}

\begin{abstract}
The present-day compressors development is a labor-intensive problem, because compressor structure should meet different requirements to the design characteristics. It's reasonable to find optimal combination of compressor design parameters using the mathematical optimization resources. In this paper the multi-criteria optimization of the rotor and stator blades of the experimental compressor stage NASA Rotor 37 is carried out. The goal of this work is the analysis of different blade parameterization schemes and determination of optimum number of variable parameters for compressor stage aerodynamic characteristics improvement. As an optimization criterions efficiency of compressor stage was used. Flow rate and pressure ratio values should not exceed base values more than $\pm 0.5 \%$. In order to research an effect of the number of variables to the optimization results, the four parameterized models were created. The optimization of the NASA Rotor 37 was carried out using all created parametric models. The models are characterized by number of variables, which describe the blade pressure and suction sides. As a result of optimization the NASA Rotor 37 version was found, which provide the efficiency increasing by approximately $2 \%$ while all aerodynamic and requirements are satisfied. It was also found, that increasing of the blade profile number of variables more than 14 is not rational.
\end{abstract}

\section{Introduction}

In the design development of the compressor blade it's necessary to meet different requirements for the aerodynamic, strength, and mass characteristics of the blade. It is also essential to consider the design constraints and processing limits.

To solve this problems in this work the unified automated parameterized model is used, which was specially developed for this work. This model allows researching of the blade different parameterization schemes for aerodynamic-optimal design. In this paper according to selected criterions and restrictions some optimal parameterization schemes are chosen.

For the optimization process researchers have to have parameterized blade models, which include all possible parameters, which describe the structure to be optimized. The original blade structure parameterization is individual difficult problem [1]. The geometric model specification and number of optimization parameters are depend on the result of this problem solving: the more number of parameters, the more complicated model.

\section{Optimization object}

As an optimization object the experimental compressor NASA Rotor 37 [2] has been chosen. Figure 1 shows the compressor impeller.

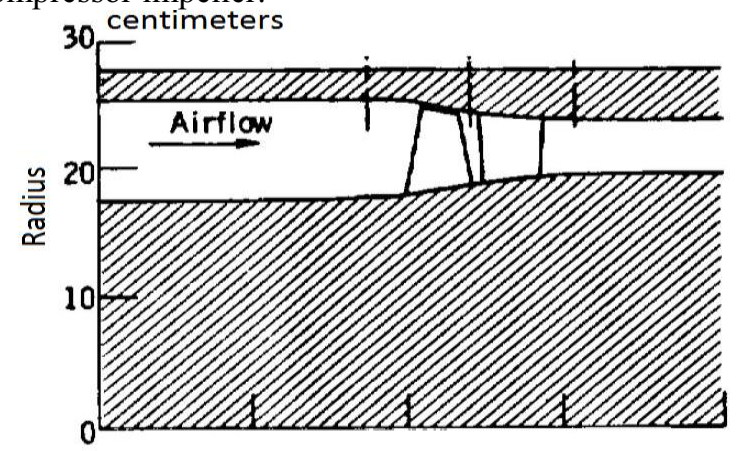

Figure 1. Experimental compressor NASA Rotor 37. [2].

The compressor parameters are represented in Table 1

Table 1. NASA Rotor 37 Parameters

\begin{tabular}{|l|l|l|}
\hline \multicolumn{1}{|c|}{ Parameter } & \multicolumn{1}{c|}{ Units } & \multicolumn{1}{c|}{ Value } \\
\hline Pressure ratio & - & 2,05 \\
\hline Adiab. Efficiency & - & 0,84 \\
\hline Flow rate & $\mathrm{kg} / \mathrm{sec}$ & 20,74 \\
\hline Rotor blades number & & 36 \\
\hline Stator blades number & & 46 \\
\hline Rotation speed & $\mathrm{rpm}$ & 17188 \\
\hline
\end{tabular}




\section{The IOSO optimization software}

The optimal projects search can be done using various numerical optimization methods. The choice of a numerical method depends on the number of variable parameters, the nature of the estimated relationships between the variable parameters, criteria, and constraints, as well as the number of set criteria and restrictions $[3,4]$.

In this work, the optimal structure search was carried out using modern multi-criteria optimization package IOSO NM [5,6], developed for solving constrained and unconstrained optimization complex one-criterion and multi-criteria problems for objective functions of different categories.

IOSO NM uses the method of indirect statistical optimization based on self-organization. This method is based on the application of response surface creation technology for the approximation of the objective function and the restrictions. IOSO algorithms have good invariant properties and a high level of stability; they allow extremum seeking when there are noncomputability domains during the optimization of compound objects.

For the evaluation of optimization method performance the optimization numerical experiments test was carried out. The results show that for smooth test functions IOSO algorithms are no worse than well-known gradient algorithms. But for composite objective function with non-computability domains, discontinuities, and several extremums (that are more probably in practice) IOSO effectiveness has an insignificant decrease when the gradient algorithms become inapplicable.

\section{The parametric computational model}

The aerodynamic model contains the grid model of the compressor, computational domains, analysis method, and boundary conditions.

For the mesh sensitivity researches the set of computational grids with different number of elements was created: Mesh1 (1,04 million cells), Mesh2 (1.84 million cells), Mesh3 (3,13 million cells).

The computational grid on the blades surfaces (Mesh2) is illustrated on Figure 2.

The aerodynamic research was carried out using Numeca FineTurbo within the limits of RANS with the turbulence model Spalart-Allmaras.

Calculations are performed for one operation mode of the compressor, rotational speed $n=17188 \mathrm{rpm}$.

As a boundary condition at the inlet of the computational domain full flow parameters are used, after the compressor- static pressure is specified.

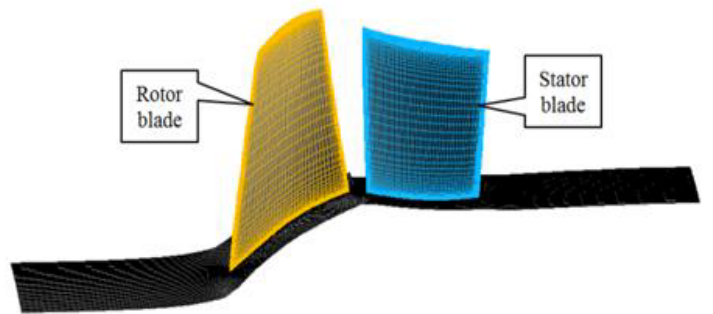

Figure 2. Computational grid on the blades.
The computational grid model of the compressor (Mesh2) is shown in Figure 3.

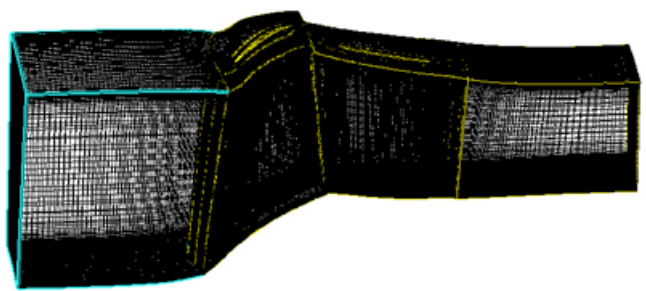

Figure 3. Compressor computational grid model.

The NASA Rotor 37 stage aerodynamic characteristics, obtained by using described computational grids in comparison with experimental characteristics [2] are shown in Figure 4.

Characteristics shows the stage pressure ratio $\pi_{k}^{*}$ versus flow rate $\mathrm{m}$ (relative to the choking point flow rate) and efficiency $\eta_{k}^{*}$ versus flow rate $m$ (relative to the choking point flow rate).
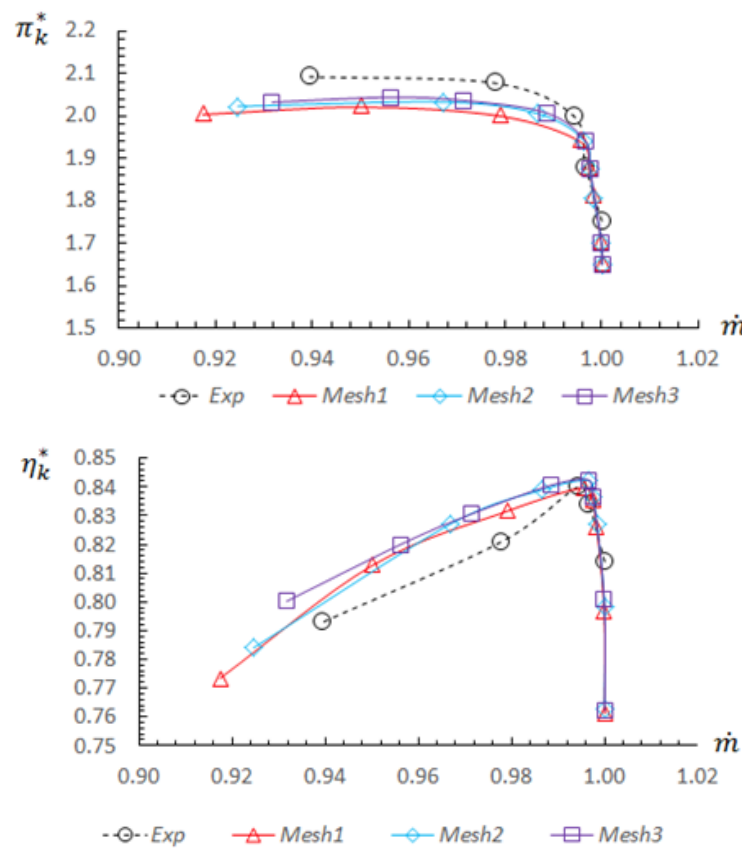

Figure 4. Comparison of calculated and experimental characteristics.

As can be seen from the illustration, the characteristics obtained match the experiment.

For the point of the characteristic with maximum of efficiency the difference between the stage parameters and experiment was calculated by formula:

$$
\delta_{j i}=\left|\frac{j_{\operatorname{mesh~} i}}{j_{\text {exw }}}\right|,
$$

where $j_{\text {mesh } i}-\mathrm{j}$-parameter value in the point with maximum of efficiency, obtained by using of the grid;

$j_{e x p}-$ value of parameter $j$ in the experimental point with maximum of efficiency;

$j$ - parameter name (compressor stage pressure ratio $\pi_{k}^{*}$, flow rate $m$, compressor stage efficiency $\eta_{k}^{*}$ ). 
Figure 5 shows the difference between the stage parameters and experiment versus used computational grid.

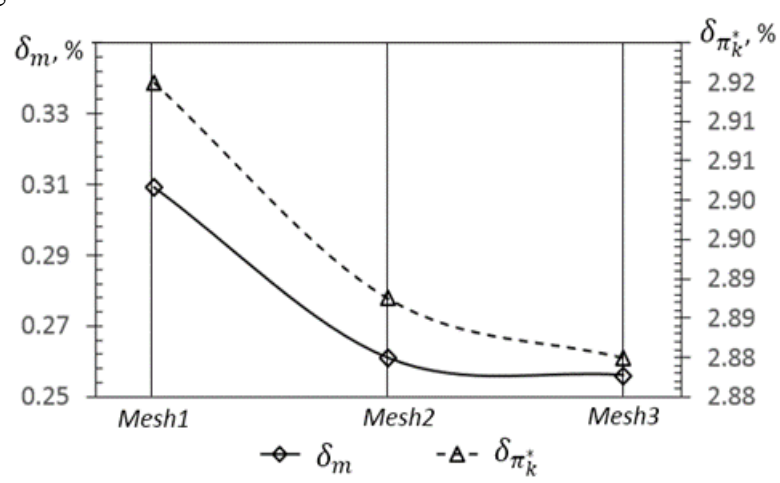

Figure 5. The difference between the stage parameters and experiment.

The difference between calculation and experiment for $\eta_{k}^{*}$ was the same for all grids $(0,29 \%)$.

The difference between calculation and experiment for $\delta_{m}$, calculated using Mesh1 and Mesh2 is approximately 0,024, while using of Mesh2 и Mesh3 - approximately 0,005 . The difference for $\delta_{\pi_{k}^{*}}$, calculated using Mesh1 and Mesh2 is approximately 0,028 , while using of Mesh2 and Mesh3 - approximately 0,008 .

In this case the best grid is Mesh3, but it's the biggest grid, so using of this grid could significantly increase the timing of the optimization. Thus, Mesh2 was chosen for the optimization.

Aerodynamic characteristics analysis showed, that the stage efficiency matches the experiment. But the level of this value may be increased.

\section{The blade parameterization schemes used in the optimization}

For the optimization of the blade design it's necessary to have parameterized model, which allows to generate blade structure based on some set of variables. The type and the number of variables in the parameterized model of the blade could be different.

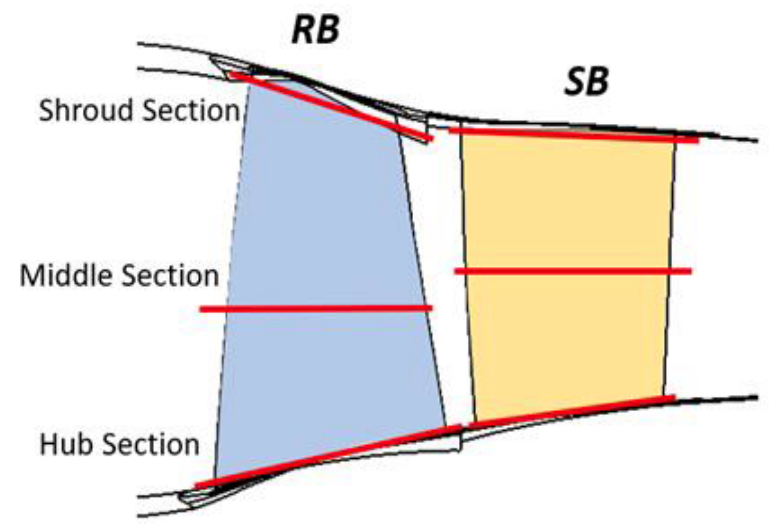

Figure 6. NASA Rotor 37 parametric sections location scheme.

Although the number of variables could significantly affect to the optimization results, there are no recommendations about what number of parameters is most rational. The most rational number of variables is the minimal number of variables, which allows to obtain the best improving of the goal function with minimal number of calls to the mathematical model.

In this work the compressor rotor and stator blades parametric models were made using Numeca AutoBlade software.

2D-profiling of each blade was performed in three sections: hub section $(\mathrm{H}=0.1)$, middle section $(\mathrm{H}=0.5)$ and shroud section $(\mathrm{H}=0.9)$ (Figure 6).

In this paper the following parameterization scheme was chosen.

The midline was specified as Bezier spline, which has three control points. The position of control point is determined by the blade angles $\beta_{1}$ and $\beta_{2}$, the profile stagger angle $\gamma$ and the chord length (Figure 7).

Suction and pressure side shape was determined by Bezier curves with several poles, leading and trailing edge radiuses and trailing edge wedge angle (Figure 8 ).

For the optimization four parameterized models of the rotor blade were created. For models creation we use the scheme described before and different number of control poles (Table 2).

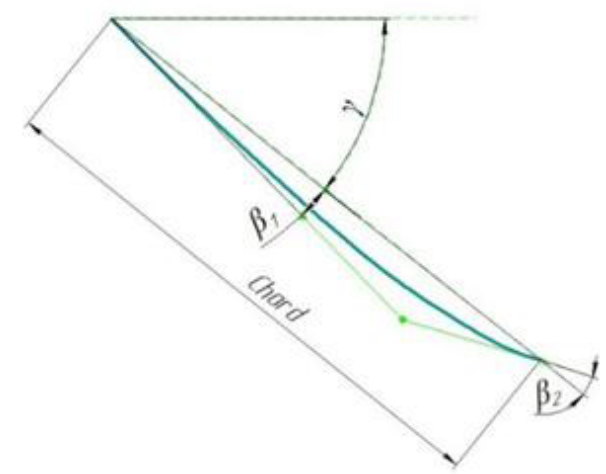

Figure 7. The blade midline in the parameterization scheme.

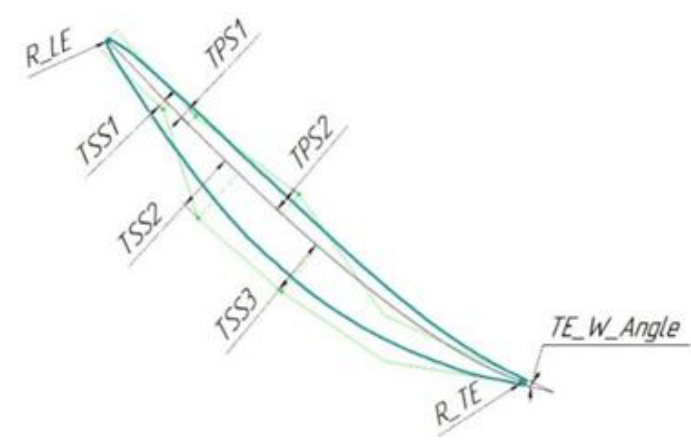

Figure 8. The pressure side and suction side curves in the parameterization scheme.

Table 2. Parameterization Models

\begin{tabular}{|c|c|c|c|c|}
\hline \multirow{2}{*}{$\begin{array}{c}\text { Mo- } \\
\text { del }\end{array}$} & $\begin{array}{c}\text { Pressure } \\
\text { side }\end{array}$ & $\begin{array}{c}\text { Suction } \\
\text { side }\end{array}$ & $\begin{array}{c}\text { Profile } \\
\text { variab- } \\
\text { les }\end{array}$ & $\begin{array}{c}\text { Total } \\
\text { number of } \\
\text { variables }\end{array}$ \\
\hline 1 & 2 & 3 & 7 & 30 \\
\hline 2 & 2 & 3 & 12 & 36 \\
\hline 3 & 3 & 4 & 14 & 42 \\
\hline 4 & 5 & 6 & 18 & 54 \\
\hline
\end{tabular}


All variables are shown on Figure 7, 8. The poles coordinates are denoted by TPS1, TPS2, TSS1...TSS3. The other suction side and pressure side poles coordinates were automatically computed by Numeca AutoBlade based on information about the values of chord, leading edge radius $R \_L E$ and trailing edge radius $R \_T E$, wedge angle TE_W_Āngle.

The model\#1 has the same number of control points as the model $\# 2$, but the pressure side and suction side shape in model \#1 were fixed, and the rest of the variables were changed.

Parameterized model of the stator blade was identical in all cases. The stator blade was changed by only angles $\beta_{1}$ and $\beta_{2}$ and stagger angle $\gamma$. This approach was chosen for decreasing of total number of variables in optimization problem.

\section{The fitting process}

The blade parameterized models were created using Numeca AutoBlade software by parameters shown in Table 2. In order to obtain the same shape of the parameterized model as the original one, in Numeca AutoBlade software there is the fitting process. During this fitting process the values of variables are chosen automatically for the purpose of minimization the parameterized blade shape deviation from original structure shape. Advantage of this fitting-technology is automating creation of parameterized model for the specified type and number of variables. Disadvantage of this technology is the fact that the base blade shape may not be accurate obtained for the parameterized blade. This situation leads to the fact, that aerodynamic parameters of the parameterized blade and the original blade will be different.

\section{The fitting effect: the aerodynamic characteristics change}

Figure 9 shows the comparison of the base compressor aerodynamic parameters and the compressor obtained by the fitting procedure for all the models described before. The model\#1 is obtained from model\#2, so the values for them are the same.

$\pi \mathrm{K}$

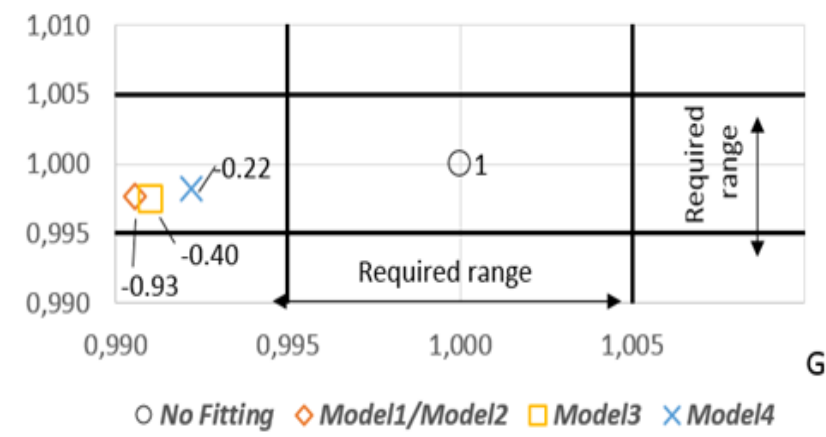

Figure 9. Compressor parameters before and after fitting.

The pressure ratio here is set as one. Required parameters range $\pm 0,5 \%$ from the base value.
The relative values of the pressure ratio are indicated by markers. The subscripts under the markers show the relative compressor efficiency decreasing in comparison with the original one. The lines in Figure 9 show the limits of working point location in the optimization process.

The analysis of Figure 9 shows:

- Chosen parameterization models didn't satisfied the flow rate limits. At the same time all of models satisfied the pressure ratio limits.

- The parameterization model with maximum of variables (model\#4) provided the flow rate decreasing by $0.77 \%$ while relative $\pi_{\mathrm{K}}$ is decreasing by $0.17 \%$ and relative efficiency decreasing by $0.22 \%$.

- The parameterization model with minimum of variables (model\#2) provided decreasing of the flow rate relative to the base value by $0.93 \%$ while relative $\pi_{\mathrm{K}}$ decreasing by $0.24 \%$ and relative efficiency decreasing by $0.93 \%$.

After the fitting-process it was found, that all the parameterized models didn't satisfied the specified limits. So, the optimization problem was divided into two stages:

- Optimization for all aerodynamic limits satisfaction for each parameterized model.

- Optimization for maximum possible increasing of goal functions (efficiency maximization) for each parameterized model.

\section{The blade optimization process}

As an optimization module IOSO software is used. Each optimization iteration consists of several steps:

- Forming a vector of variable parameters in IOSO software, transfer them to the used mathematical model;

- Mathematical model start: consecutive modification and analysis of used models, the calculation of criteria and restrictions;

- Forming of an output parameters list, transfer them into IOSO software;

- Analysis of the obtained data in IOSO software, convergence check:

- if there is no convergence, the process goes to step №1, i.e. IOSO software forms a new list of variable parameters, which is passed into the model;

- if there is a convergence, the optimization process stops.

Optimization is performed on the four PC with the following characteristics: 16GB of RAM, 6 cores $3.0 \mathrm{GHz}$.

The time of one iteration is about 42 minutes: 1-2 minutes for strength analysis and 40 minutes for aerodynamic analysis.

\section{The optimization. stage 1}

The goal of the first stage of the optimization is to gain all aerodynamic limits satisfaction for each parameterized model. 
The first stage optimization criterions are:

- Flow rate increasing;

- Efficiency increasing;

The first stage optimization restrictions are:

- Flow rate change relative to the base one $1 . .+0,5 \%$

- Pressure ratio change relative to the base value $\pm 0,5 \%$.

The problem was considered as solved when it was found the point, which met flow rate limits $\pm 0,5 \%$ from the base value.

The results of first stage optimization problem is shown in Figure 10.

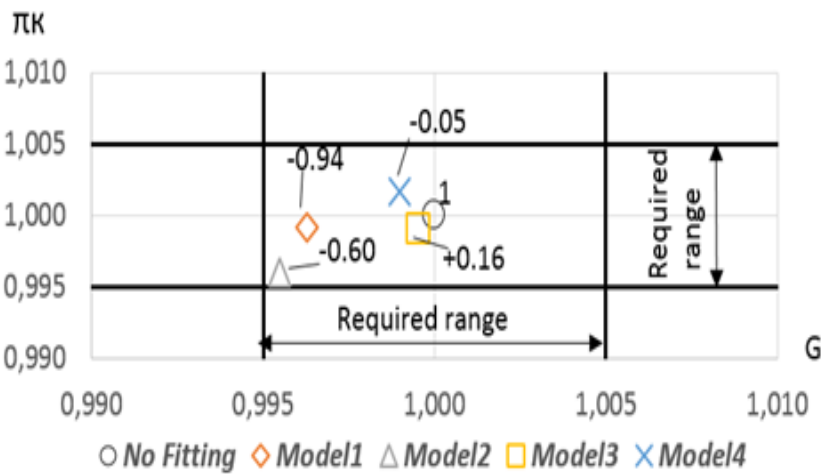

Figure 10. Parameters of the parameterized models after the first stage of optimization.

The relative values of the pressure ratio are indicated by markers. The subscripts under the markers show the relative compressor efficiency changes in comparison with the base values.

The number of calls for each model is shown in Table 3.

As can be seen from Figure 10, all obtained blade models satisfy all the required flow rate and pressure ratio parameters.

As a result of the first stage of optimization:

- The most rapid solution was obtained using the model\#2 (with minimum number of points on pressure side and suction side) in 26 calls to the model, but there is significant efficiency decreasing by $0.94 \%$ in comparison with base value;

- The solution with maximum efficiency increasing $(+0.16)$ was obtained using model\#3 (with middle number of points) in 83 calls;

- Using of model\#1 (with fixed blade thickness) asked for 170 calls and efficiency decreasing was approximately $0.6 \%$ in comparison with base value.

All points found were used as initial solutions for the second stage of optimization.

Table 3. Number of Calls to the Model

\begin{tabular}{|c|c|}
\hline Model & Number of calls \\
\hline 1 & 170 \\
\hline 2 & 26 \\
\hline 3 & 83 \\
\hline 4 & 67 \\
\hline
\end{tabular}

\section{The optimization. stage 2}

The goal of the second stage of the optimization is to gain maximum increase of goal functions for each parameterized model.

The second stage optimization criterions were:

- The stage efficiency increasing;

The second stage optimization criterions are:

- Flow rate change relative to the base one $-1 . .+0,5 \%$;

- $\quad$ Pressure ratio change relative to base value $\pm 0,5 \%$;

As a variables the parameters of parameterized models from Table 2 were used.

The problem was considered as solved when the IOSO software will call the model 2000 times.

The more model variables used the more efficiency we can get. The goal functions maximum values relative to the base value are shown in Table 4.

Table 4. The Goal Functions Changes

\begin{tabular}{|c|c|}
\hline Model & Rel.max eff, \% \\
\hline 1 & 0.65 \\
\hline 2 & 1.44 \\
\hline 3 & 2.11 \\
\hline 4 & 2.15 \\
\hline
\end{tabular}

Table 4 shows that the maximum efficiency increasing is obtained using the model\#4 (with big number of variables). The best compromise was found using the model\#3 (with middle number of points).

In order to compare the optimization criterions improving dynamics for different models, Figure 11 shows the efficiency improving history.

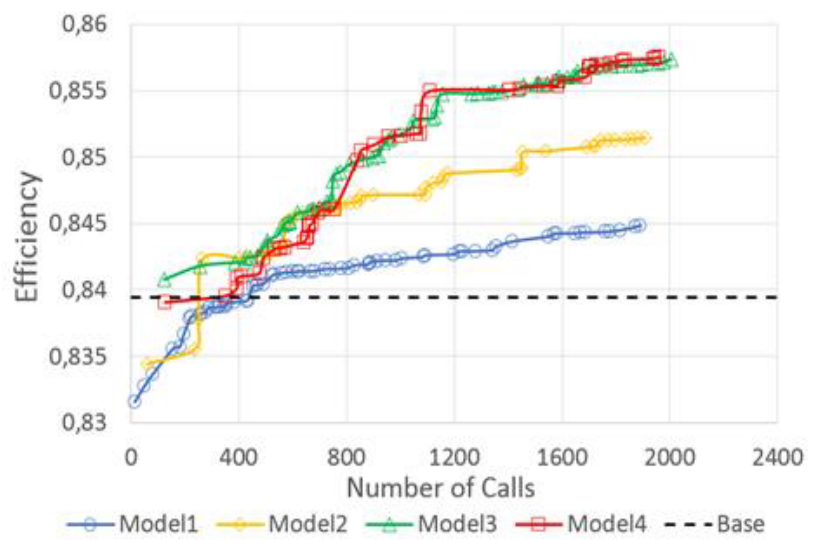

Figure 11. The efficiency improving history for each model.

The analysis of Figure 11 shows:

- Using of model\#1 gives minimal efficiency increase in 2000 calls;

- Model\#2 gives efficiency increase by $1.4 \%$ in comparison with base value in 2000 calls;

- Model\#3 and model\#4 gives approximately equal efficiency increases by $2.1 \%$

- All models shows equal efficiency increase before 750 calls. 


\section{The geometrical models changes analysis}

For the point with maximum of efficiency the analysis of geometrical models changes for all models was carried out.

Figure 12 shows the profiles camber distribution for all models in a point with maximum efficiency increase in 2000 iterations.

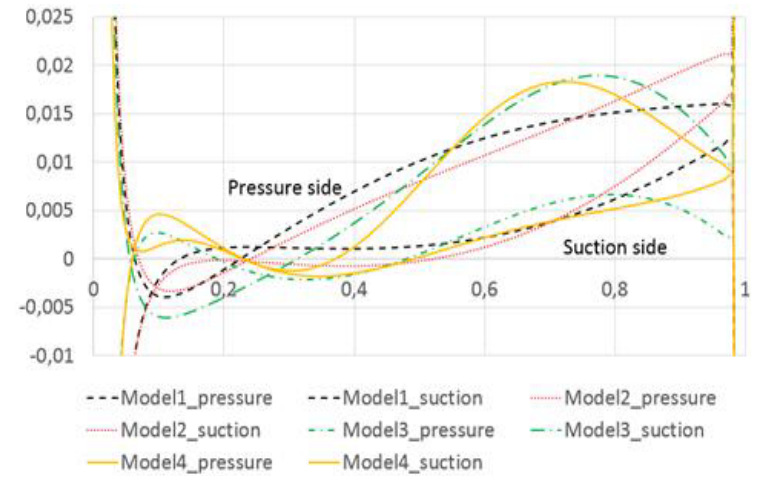

Figure 12. The profiles camber distribution for all models.

As can be seen from the Figure 12, the maximum number of bends on the pressure side and suction side of the blade is 3 . That means that for the profile shape modeling it's enough to use the model\#3 with seven variable points.

For each model for the point with maximum of efficiency the analysis of geometric parameters (common for all the schemes): chord, $\gamma, \beta_{1}, \beta_{2}, \mathrm{R} \_\mathrm{LE}, \mathrm{R} \_\mathrm{TE}$, TE_W_Angle was carried out by formula:

$$
\delta_{j}=\frac{1}{a} \sum_{i=1}^{a}\left|\frac{\delta_{j i \text { naw }}}{\delta_{j i \text { bara }}}-1\right| \cdot 100
$$

where: $\delta_{j}-$ total average deviation of the geometric parameter;

$\delta_{\text {ii new }}$ - the deviation of geometric parameter $\mathrm{j}$ in the section $i$ for the optimized structure;

$\delta_{i \mathrm{i} b a s e}$ - the deviation of geometric parameter $\mathrm{j}$ in the section $i$ for the base structure.

Figure 13 shows the values of $\delta_{i}$ for each model.

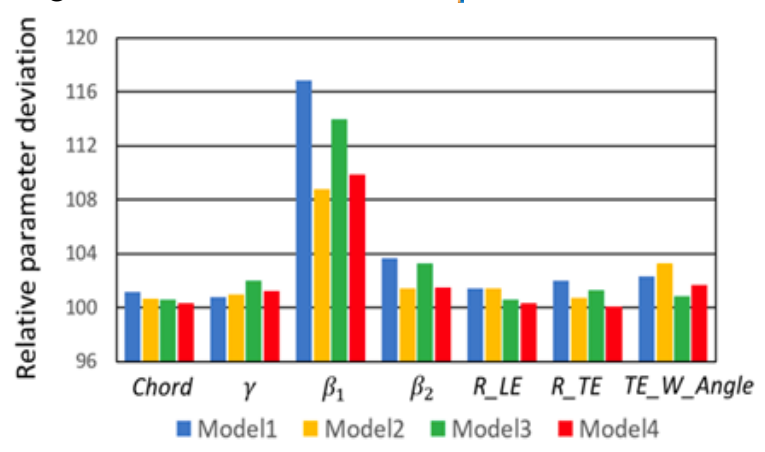

Figure 13. The geometric parameters relative deviation for each model in the point with maximum of efficiency.

Figure 13 shows that the maximum changes of geometric parameters take place for parameter $\beta_{1}$ for each model. For model\#1 total changes of the parameter $\beta_{1}$ is $17 \%$. It also can be seen that parameters Chord, R_LE_T,
R_TE, which mostly effect to the area of the sections, has been significantly changed for the model\#1. So, that fact can explain the largest decrease of the centrifugal force in the model\#1.

Figure 14 shows the cross sections of the original and optimized blades near the hub $(\mathrm{H}=0.1)$, in the middle $(\mathrm{H}$ $=0.5)$, and near the periphery $(\mathrm{H}=0.9)$ for model $\# 3$.

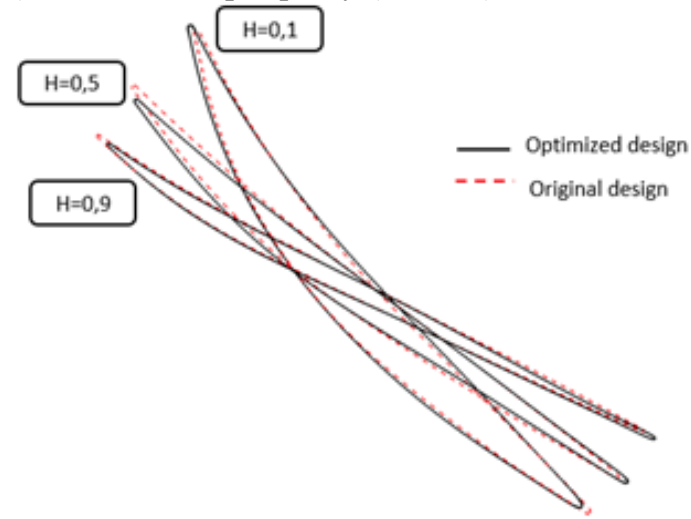

Figure 14. The cross sections of the original and optimized blades in three sections.

Figure 15-18 shows the aerodynamic research results of the base (original) and optimized compressor stage Rotor37 as Mach number fields. The changes are highlighted with the blue frame.

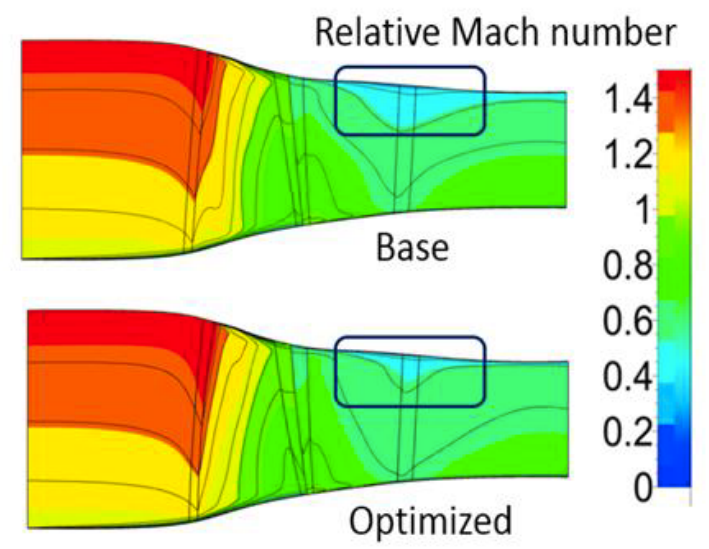

Figure 15. Mach number fields in base and optimal compressor stage in a meridional section.

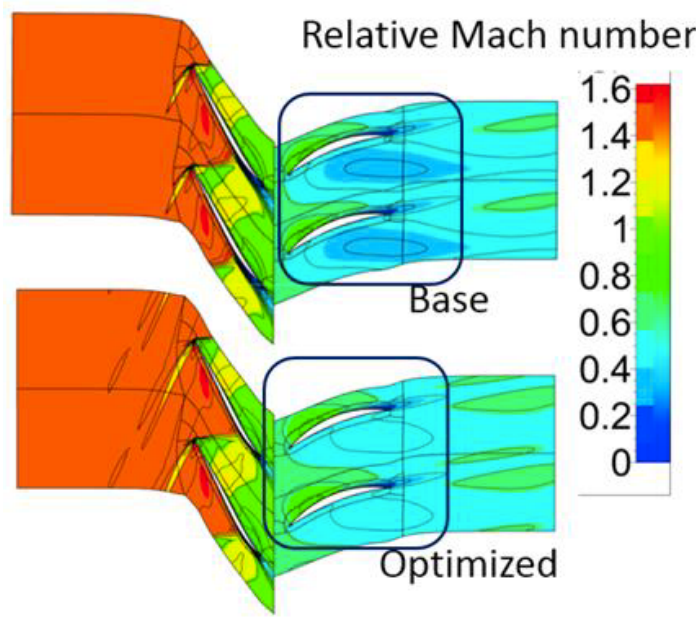

Figure 16. Mach number fields in base and optimal compressor stage in a peripheral section $(\mathrm{H}=0.9)$. 


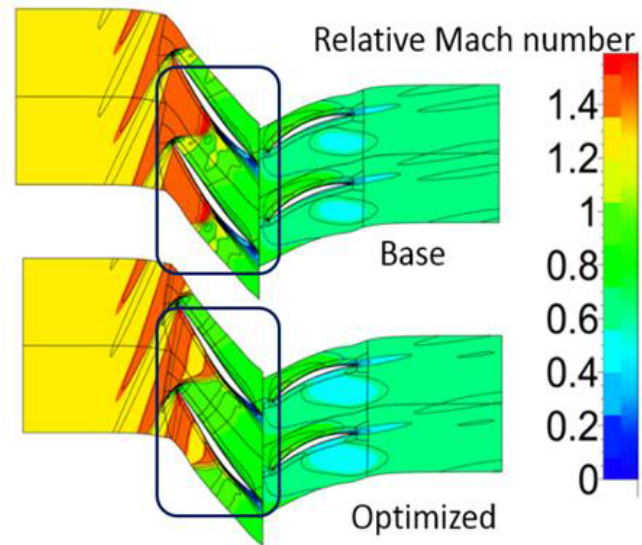

Figure 17. Mach number fields in base and optimal compressor stage in a middle section $(\mathrm{H}=0.5)$.

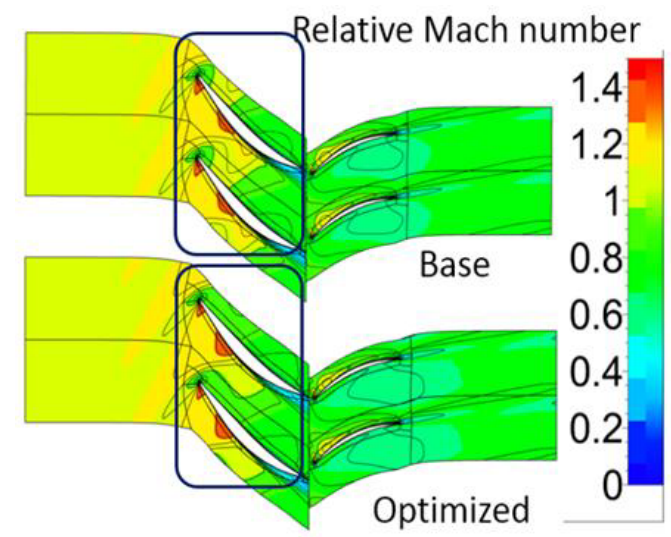

Figure 18. Mach number fields in base and optimal compressor stage in a hub section $(\mathrm{H}=0.1)$.

As can be seen in Figure 15-18 the reasons of the efficiency increase of the optimized version of Rotor37 are the flow separation reduce in a peripheral section of the stator blade (Figure 16) and the decrease of the intensity of the shock waves in the middle and hub sections of the rotor blade (Figure 17, 18).

\section{Conclusion}

The multidisciplinary optimization of the compressor stage Rotor37 was carried out. During the optimization process a unified parameterized 3D model, which describes all the necessary computational domains, was used. The aerodynamic calculation was carried out based on the flow model, which provides the automatic modification of the rotor and stator blades.

For this research several compressor rotor blade parameterized models were made. Each model has its own number of variables, which describe the pressure and suction sides of the blade. Parameterized models were made using Numeca AutoBlade software and "fitting".

It was found, that increasing of the number of variables on the profile sides leads to the fact, that the parameterized model work parameters deviation from the basic model parameters is reduced. That means that the more detailed model, the better it describes the original structure.

However, no model ensured that the flow rate determined using the parametric model satisfy the limits of $\pm 0.5 \%$ of the base value.

The results of the described optimization first stage, which meet all aerodynamic requirements, were used as initial points for the second step of the optimization.

At the second stage, for each model, the optimization task was solved for 2000 calls. The optimization criterions was the efficiency increasing.

As a result, the compressor stage Rotor37 version was found, which provide the efficiency increasing by $2.11 \%$ and blade centrifugal force decreasing by $8.96 \%$.

It was found, that increasing of the number of points describing the shape of the profile more than 14 (Model\#3) is not appropriate, since this does not lead to the efficiency maximum improvement.

The simplest model\#1 (where the blade thickness is fixed) has the smallest number of variables, but provides an efficiency increase by $1.45 \%$ less than model\#3.

Thus, the most rational was the model\#3, where the shape of the pressure side and the suction side of the profile is described by 14 variables. Using of this scheme allows to obtain the maximum increase in efficiency with the minimum number of variables.

The optimization process was completely automated and took place without user participation.

\section{References}

1. I. Arsenyev, F. Duddeck and A. FischersworringBunk, Proc. ASME Turbo Expo, (2015)

2. L.Reid and R.D.Moore, NASA Technical Paper 1337, 122, (1978)

3. I.M. Sobol and R.B. Statnikov, Choosing the optimal parameters in problems with many criteria, 175, (2006)

4. I.N. Egorov, G.V. Kretinin, I.A. Leshchenko and S.V. Kuptzov, Proceedings of WCSMO6 Symposium, (2005)

5. I.N. Egorov, G.V. Kretinin, I.A. Leshchenko and S.V. Kuptzov, In Proc. of 10th AIAA; ISSMOMultidisciplinary Analysis and Optimization Conference, (2004)

6. I.N. Egorov and Kretinin, G.V., Proc. ASME Turbo Expo, (2007) 